\title{
Editorial
}

\section{Deep Learning for Remote Sensing Image Understanding}

\author{
Liangpei Zhang, ${ }^{1}$ Gui-Song Xia, ${ }^{1}$ Tianfu Wu, ${ }^{2}$ Liang Lin, ${ }^{3}$ and Xue Cheng Tai ${ }^{4}$ \\ ${ }^{1}$ State Key Lab. LIESMARS, Wuhan University, Wuhan 430079, China \\ ${ }^{2}$ Department of Statistics, University of California, Los Angeles (UCLA), Los Angeles, CA 90095, USA \\ ${ }^{3}$ School of Advanced Computing, Sun Yat-Sen University, Guangzhou 510275, China \\ ${ }^{4}$ Department of Mathematics, University of Bergen, 5020 Bergen, Norway
}

Correspondence should be addressed to Liangpei Zhang; zlp62@whu.edu.cn

Received 28 June 2015; Accepted 28 June 2015

Copyright (C) 2016 Liangpei Zhang et al. This is an open access article distributed under the Creative Commons Attribution License, which permits unrestricted use, distribution, and reproduction in any medium, provided the original work is properly cited.

Deep learning has become one of the most important breakthroughs in artificial intelligence over the past decade. Deep learning contains a variety of methods, including neural networks, hierarchical probabilistic models, and many specific unsupervised and supervised feature-learning algorithms. The biggest difference between deep learning and the classic visual recognition methods is that deep learning methods automatically learn features from a huge amount of data, rather than requiring engineering features by hand. Therefore, we can conveniently learn good features for new specific tasks without much expertise and effort of designing features. In addition, deep learning approaches make better use of big data and provide an end-to-end learning framework in which jointly learning feature transformations and classifiers via the back propagation technique makes their integration optimal.

Deep learning is about learning hierarchical feature representations. Deep architectures with multiple levels attempt to learn hierarchical structures and seem promising in learning simple concepts first and then successfully building up more complex concepts by composing the simpler ones together. It accords with human's visual cognition of learning abstract concepts on top of less abstract ones. These highlevel feature representations are more powerful and robust in typical visual tasks.

In the intelligent interpretation of remote sensing images, the automatic target detection (or recognition) and highresolution remotely sensed image classification are two hot topics, and both of these two tasks are carried out by first computing the low-level features in the raw images. For different kinds of remote sensing images (e.g., SAR images and hyperspectral images), the corresponding specific feature representations are available. Through applying deep learning methods, we are free of these handcrafted low-level features and can automatically learn mid-level and higher-level ones from a large amount of unlabeled raw samples beyond the types and domains of remote sensing images. Deep leaning methods can undoubtedly offer better feature representations for the related remote sensing task, and there is a bright prospect of seeing more and more researchers dedicated to learning better features for the target detection and scene classification tasks by utilizing deep learning methods appropriately.

This special issue concentrates on the research in new methods, algorithms, and architectures of deep learning to handle the practical challenges in remote sensing image processing. The papers in this issue can be roughly organized into three main categories: (a) remote sensing imagery classification, (b) change detection of multitemporal remote sensing images, and (c) fusion of diverse types of images.

Papers in category (a) deal with the classic classification problem for distinct types of remote sensing images. The paper by Z. Huang explores the synergetic neural networks optimized by an improved quantum particle swarm algorithm for mangroves classification. The paper by Q. Lv et al. introduces deep belief networks to extract effective contextual mapping features for the task of PolSAR image classification. The paper by $\mathrm{W}$. Hu et al. specially designs a fivelayer convolutional neural network to classify hyperspectral images directly in spectral domain. The paper by D. Lin et al. proposes a novel multifeature fusion strategy via the sparse 
canonical correlation analysis and Bayesian information criterion for high-resolution satellite image classification.

Paper by L. Huang et al. introduces the 2D-Otsu threshold segmentation methods to analyze the changing area between two temporal remote sensing images, which belongs to category (b). Category (c) also contains one paper by X. Zhang et al., which presents novel methods for automatic fusion of 2D hyperspectral images and 3D laser scans by exploring collinearity equation and direct linear transformation to create the initial corresponding relationship of the two raw datasets.

Note that this special issue only provides a small number of papers and relatively narrow insights on remote sensing image understanding, and it is impossible to cover complete aspects of remote sensing applications with deep learning methods due to the page limitations.

\title{
Acknowledgment
}

Many individuals made contributions to the success of this special issue. We appreciate all the authors for their submissions, as well as all the reviewers for their careful and professional review.

\author{
Liangpei Zhang \\ Gui-Song Xia \\ Tianfu $\mathrm{Wu}$ \\ Liang Lin \\ Xue Cheng Tai
}




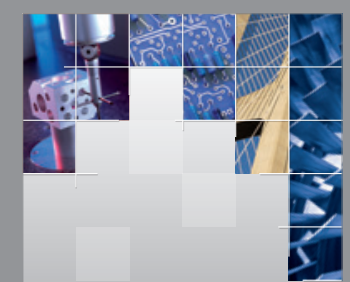

\section{Enfincering}
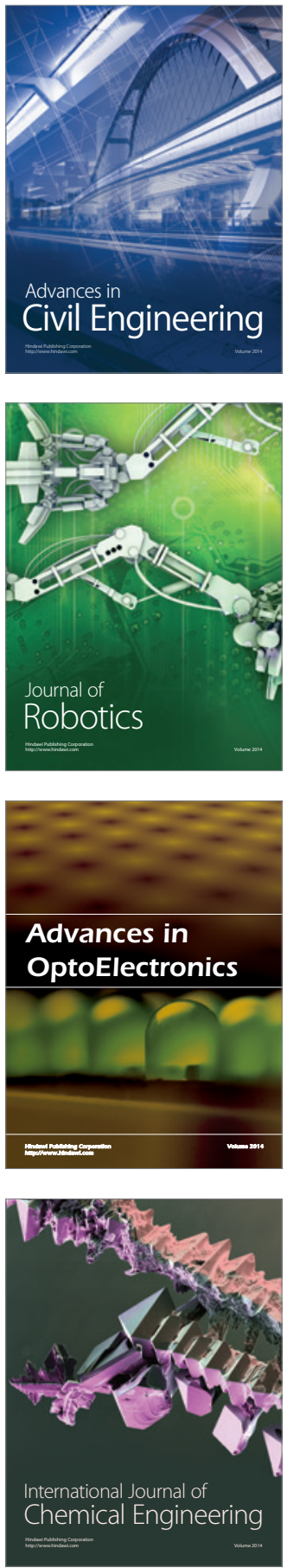

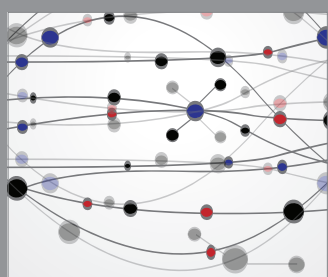

The Scientific World Journal

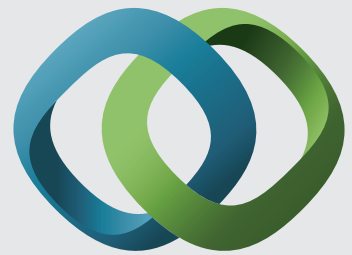

\section{Hindawi}

Submit your manuscripts at

http://www.hindawi.com
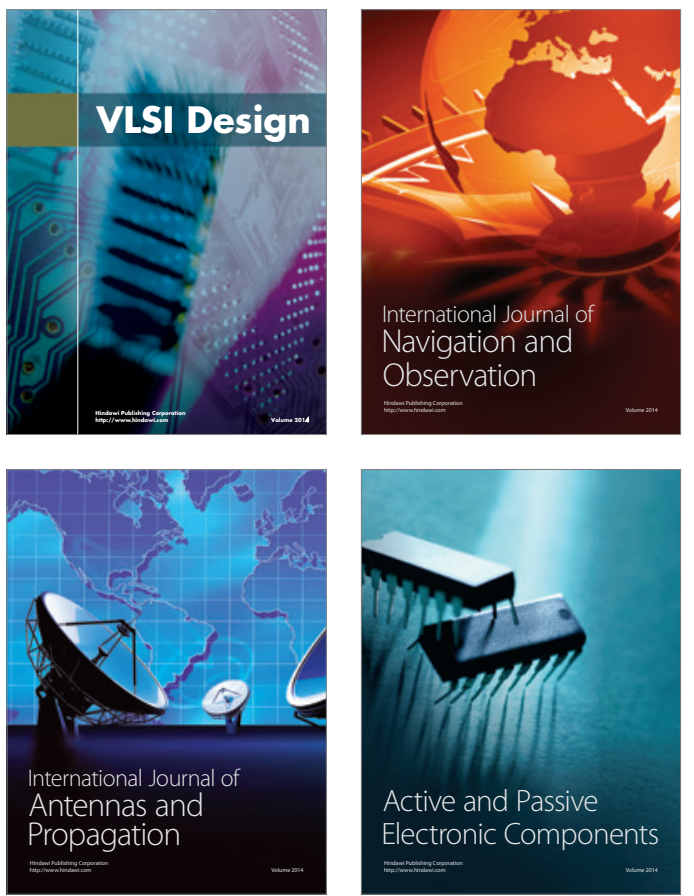
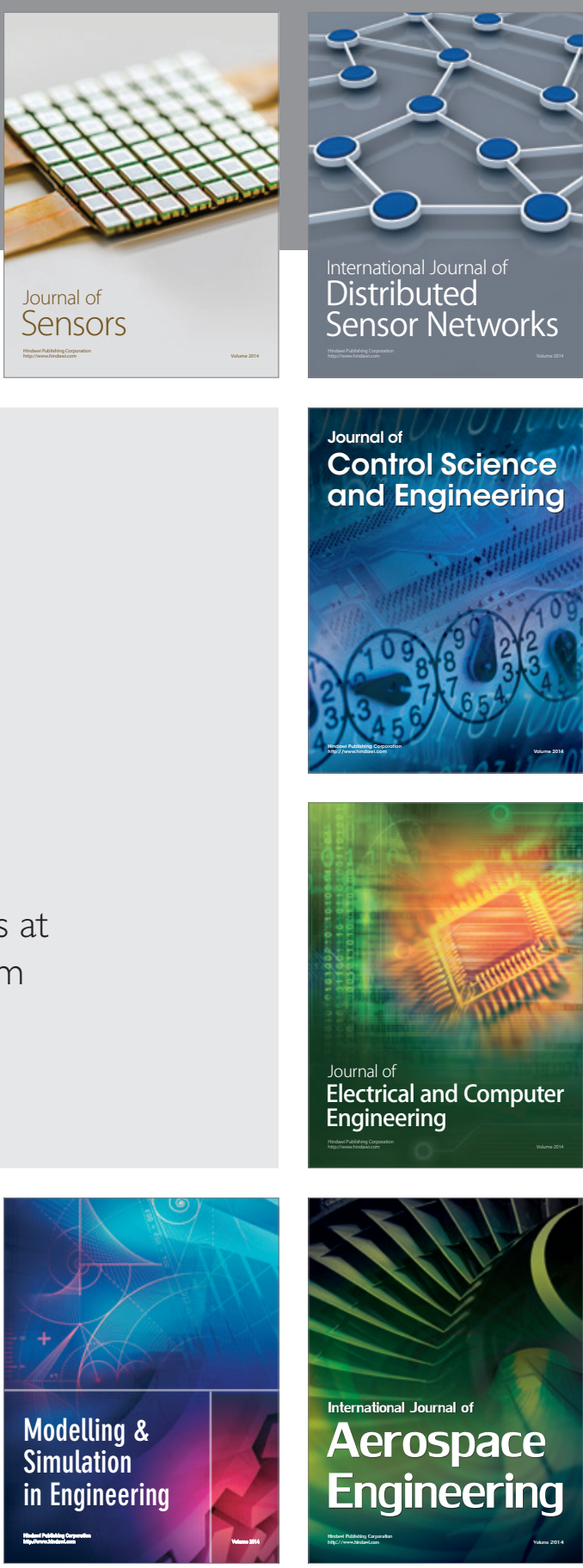

International Journal of

Distributed

Sensor Networks

Journal of

Control Science

and Engineering
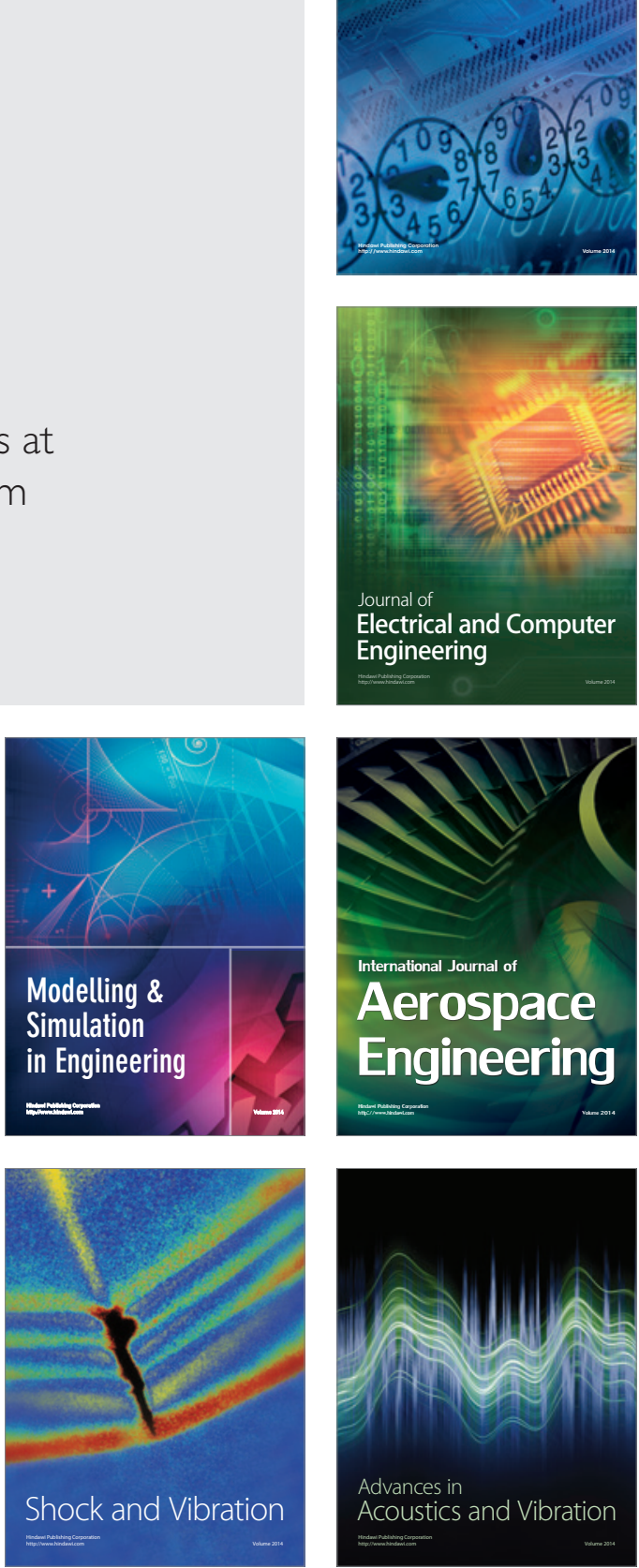